POS $\quad \begin{gathered}\text { PROCEEDINGS } \\ \text { OF SCIENCE }\end{gathered}$

\title{
Error Field in Tokamaks: a Plasmaless Measurement Approach
}

\section{Andrea Gaetano Chiariello}

\section{Alessandro Formisano'}

\section{Raffaele Martone}

Dipartimento di Ingegneria Industriale e dell'Informazione, Seconda Univ. di Napoli

Via Roma 29, Aversa (CE), Italy

E-mail: AndreaGaetano.Chiariello@unina2.it,

Alessandro.Formisano@unina2. it, Raffaele. Martonelunina2. it

\section{Shakeib Arshad}

Fusion for Energy

c/ Josep Pla, $n^{\circ}$ 2, Torres Diagonal Litoral

Barcelona, Spain

E-mail: Shakeib.Arshadef4e.europa.eu

\section{George Vayakis}

ITER Organization, Diagnostics Division

St Paul Lez Durance Cedex, France

E-mail: George. Vayakis@iter.org

\begin{abstract}
Due to a number of causes, including manufacturing and assembly tolerances on magnets, presence of feeders and joints, non-symmetric iron and magnet parts, discrepancies between design and actual magnetic field maps inevitably appear in Tokamaks. Such Error Fields may drive plasma to loss of stability; therefore they are carefully counteracted using suitable correction coils.

According to present specifications, error field amplitudes in ITER will be estimated by direct measurements, using magnetic sensors, with suited spatial resolution and frequency bandwidth (including MHD saddles, outer and inner vessel coils, and possibly partial flux loops). A second technique is under consideration, taking benefit of plasma response under suited excitation; this technique will not be further considered here.

The first approach is suited for plasmaless shots to be carried out after final assembly of ITER and after cool-down: taking advantage of the magnetic measurements, the actual coils shape and position can be identified, and from the estimated actual configuration the possible lack of symmetry in magnetic field is assessed.
\end{abstract}

\section{${ }^{1}$ Speaker}


Error Field in Tokamaks: a Plasmaless Measurement Approach

In the paper, a description of plasmaless measurements, and their use in the perspective of estimating EF by means of the reconstruction of the magnets deformations, will be presented and discussed.

First EPs Conference on Plasma Diagnostics - $1^{\text {st }}$ ECPD

14-17 April 2015,

Villa Mondragone, Frascati (Rome) Italy 


\section{Introduction}

Error Fields (EF) in Tokamaks are deviations from the nominal magnetic field configuration that can drive plasma to instability also with very small amplitudes, since they can induce locking in plasma rotations [1]. In order to get a synthetic measure of EF, a weighted sum of the three lower field harmonics $m=1,2,3$ and $n=1$ of the EF map can be used. The resulting figure of merit is indicated as the ' 3 -modes' error index $B_{3 \text {-mode }} / B_{0}$ :

$$
\frac{B_{3-\text { mode }}}{B_{0}}=\sqrt{W_{11}\left|\boldsymbol{B}_{1,1}\right|^{2}+W_{21}\left|\boldsymbol{B}_{2,1}\right|^{2}+W_{31}\left|\boldsymbol{B}_{3,1}\right|^{2}}
$$

where $B_{3 \text {-mode }}$ is the square averaged amplitude of the $(1,1),(2,1)$ and $(3,1)$ modes and $B_{0}(5.3 \mathrm{~T})$ is the nominal toroidal field at plasma axis. Fourier transform is performed on $q=2$ surface at start of flattop (SOF), when mode locking is most likely to take place.

A different definition of the EF figure of merit has recently been proposed [2], but it will not be considered here, since it requires measurements in presence of plasma, while just plasmaless approaches are considered in this work.

EF arise from coil deformations and misalignments and to joints, busbars, and more generally to non axisymmetric structures [3]. Note that the segmentation of toroidal field system breaks the symmetry, but, thanks to $20^{\circ}$ periodicity of TFC, this field ripple does not have a relevant impact on $\mathrm{EF}$.

The impact of EF has been extensively investigated during ITER design phase, but in the actual experimental device, EF amplitudes will be estimated from measurements. Different possibilities do exist, each suited to a particular aspect. Possible plasma-less measurements could also be used to measure deviations of the field coils from axial symmetry immediately prior to ITER's first plasma operation [4]. Similarly, time-varying error fields can be measured also by flux probes located in the inner surface of Vacuum Vessel. EF contributions due to toroidal field coils, on the other hand, cannot be evaluated by using flux probes (such as MHD saddles or PFL, or pickup coils), since those coils operate in persistent mode.

According to [5], EF amplitudes are expected to be measured using:

1 MHD saddles as primary sensors;

2 Outer vessel normal coils and outer vessel normal steady state sensors as supplementary sensors;

3 Partial Flux Loops as backup sensors.

This paper describes the assessment of EF plasmaless measurement methods, based on the uses of magnetic sensors foreseen in ITER diagnostic system, using accuracy ranges compatible with the design figures [6]. Primary sensors are first assessed to measure time-varying EF, and then the contribution of supplementary sensors, capable of measuring also low-frequency and DC components, is considered. Backup sensors are not considered here.

The analyses have been performed using specifically designed computational codes, used to assess the measurement accuracy in presence of uncertainties due to manufacturing and assembly of measurement systems (systematic errors) and in presence of disturbances due to intrinsic measurement process (e.g. measurement noise). 


\section{EF Analysis Procedure}

The amplitudes of modes involved in EF definition can be determined by the Fourier analysis of the magnetic field measured by flux and field probes located around the vessel, in a number of poloidal planes high enough to guarantee correct estimation of modes with toroidal index $n=1$. In order to assess the performance of primary and supplementary sensors in estimating EF Fourier coefficients, 2200 "actual" field maps, generated by deformed poloidal field coils, central solenoid and, limitedly to static field analyses, toroidal field coils, with manufacturing and assembly errors within tolerances as reported in [3], have been considered. Out of the total 2200, 1500 have been used to fit linear models relating measurements to single coefficients [7,8], and the remaining 700 to validate the models and assess the performance of various systems (test dataset). For such maps, estimates of coefficients provided by "misplaced" and "misaligned" and noisy sensors have been compared with the nominal ones, defining a "coefficients measurement error", and verifying if such figure is within acceptable ranges. Details of the numerical tools adopted for the analyses can then be summarized as follows:

- Single coefficients $\mathbf{B}_{\mathrm{nm}}$ are considered, taking advantage of their direct dependence from magnetic field measurements. Fourier transform is computed on a surface corresponding to $q=2$ surface at Start of Flat Top, although no plasma is present;

- The relationship among measurements and EF Fourier coefficients is assumed linear, thanks to electromagnetic field equations, and deduced from a set of randomly generated examples through correlation analysis;

- Estimation errors are computed by comparing estimated values and true values in the test dataset of an error index similar to (1), but normalized to average poloidal field $B_{p o l}$ rather than toroidal field on axis, as required in [6].

This allows using data analysis tools to assess system performance, allowing also robust data processing. The key figures required to EF measurement systems are reported in Table 1.

TABLE 1

KEY FIGURES REQUIRED IN MEASUREMENT OF ERROR FIELD/LOCKED MODE RELATIVE PERTURBATION

\begin{tabular}{|c|c|}
\hline \multicolumn{2}{|r|}{ "TECHNICAL SPECIFICATION } \\
\hline Proposed parameter: & Error field/locked mode relative perturbation $\left(\delta \mathrm{Br} / \mathrm{B}_{\theta}\right)$ \\
\hline Basic Sensor Set: & Nine arrays of In-Vessel MHD Flux Loops (AI) \\
\hline Supplementary Sensors & Outer vessel Normal field probes, either inductive (A4) or steady state (A6) \\
\hline Potential Backup sensors: & Combined In-Vessel Partial Flux Loop Sets A and B (AD) \\
\hline \multicolumn{2}{|r|}{ PROJECT REQUIREMENTS } \\
\hline Role: & 1a.1 (Machine Protection) \\
\hline Parameter range $\left(\mathrm{B}_{\mathrm{r}} /<\mathbf{B}_{\mathrm{p}}>\right)$ : & $10^{-4}-10^{-2}$ \\
\hline Time resolution: & $1 \mathrm{~ms}$ \\
\hline Spatial resolution (Wave num.): & $(1,1)<(\mathrm{m}, \mathrm{n})<(1,3)$ \\
\hline Accuracy: & $\begin{array}{l}30 \% \text { (assessed both relative error as } 30 \% \text { of Full scale: } 3 \mathrm{mT} \text {, and absolute figure } \\
\text { at lower limit: } 0.03 \mathrm{mT} \text {, considered excessive in [12]) }\end{array}$ \\
\hline
\end{tabular}

\section{Accuracy assessment for primary sensors}

The first analysis was performed by using MHD saddles alone to reconstruct the three EF coefficients using the described procedure, and then, by comparison with the original, exact data, to assess accuracy in coefficients reconstruction. The reference MHD saddles system, 55.AI [9], is depicted in Fig. 1, and its main characteristics are reported in Table 2. 
A.G. Chiariello, A. Formisano, R. Martone,

S. Arshad, G. Vayakis



Fig. 1 - MHD saddles used in this analysis (red). For the sake of comparison, also the equilibrium surface used for Fourier decomposition of field ( $q=2$ at SOF of 15MA reference inductive scenario, grey points) is reported.
TABLE 2

KEY FIGURES FOR THE MHD MEASUREMENT SYSTEM

\begin{tabular}{ll}
\hline \hline \multicolumn{2}{c}{ MHD Saddles specifications: } \\
\hline Number of Probes: & $\begin{array}{l}\text { 81 Saddles on 9 poloidal } \\
\text { sections (9 Saddles each) }\end{array}$ \\
\hline Probe type & $\begin{array}{l}\text { Inductive sensors (Flux } \\
\text { loops) }\end{array}$ \\
\hline Total Sensor Error & Relative: 0.11\%, \\
\hline $\begin{array}{l}\text { Accuracy @ 2 } \boldsymbol{\sigma} \text { for nor- } \\
\text { mal field measurement }\end{array}$ & Absolute: $2 \mathrm{mT}$ \\
\hline $\begin{array}{l}\text { Range for normal field } \\
\text { measurement }\end{array}$ & $0-2 \mathrm{~T}$ \\
\hline Nominal effective area: & $5 \mathrm{~m}^{2}$ \\
\hline \hline
\end{tabular}

In order to assesses the effectiveness of EF reconstruction from MHD signals only, the 1500 cases from the numerically generated training set were used to create a regression model, then Gaussian noise with a std. dev. of $0.1 \%$ (compatible with ITER specifications) was added, and resulting noisy signals were used to reconstruct EF. The comparison of EF index and its estimation from MHD measurements is reported in Fig. 2.

Primary sensors appear able to reconstruct EF in presence of AWGN; note that reconstruction appears the less accurate the smaller EF amplitude is.

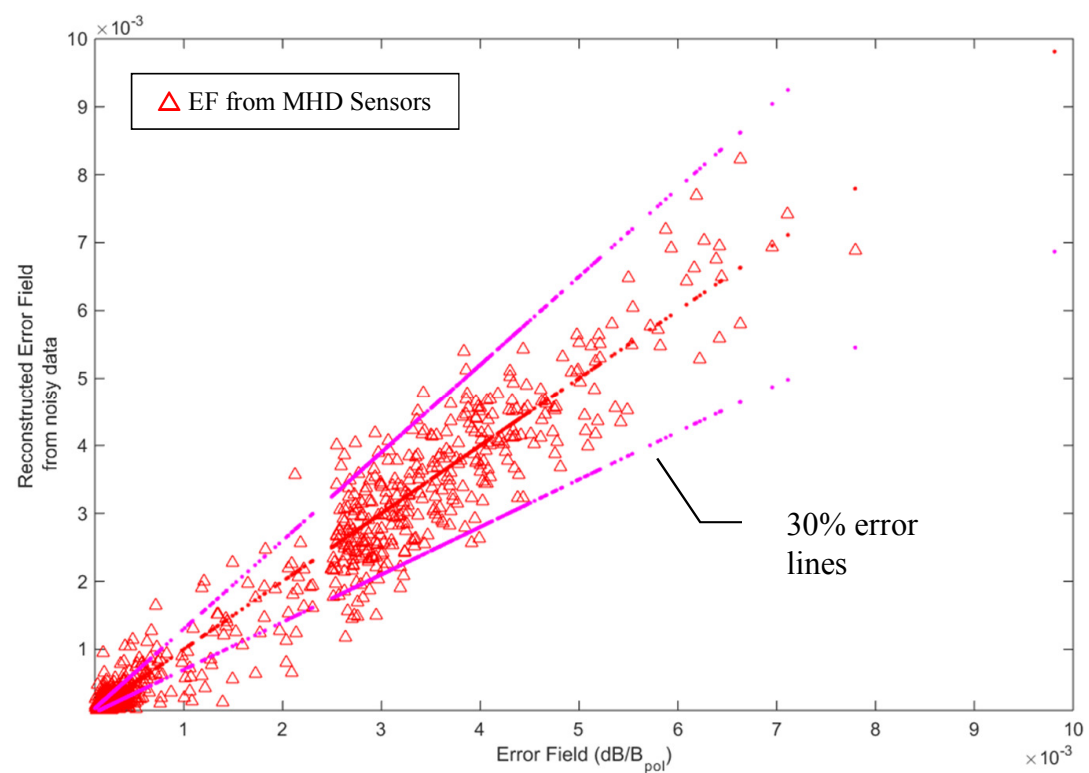

Fig. 2 - Comparison between "numerically simulated" and estimated EF amplitudes for test set. Magenta lines represent $+/-30 \%$ error levels. Only signals from primary sensors are considered.

\section{Accuracy assessment for supplementary sensors}

A second analysis was performed by adding simulated signals from supplementary sensors to improve performance of primary ones. In this case the information from supplementary sensors is used "simultaneously" with the primary ones, by adding further columns (corresponding to supplementary sensors measurements) to the model matrix, transforming probe signals to error 
field coefficients. A robust model was used in this case, as a countermeasure to face with poor performance of A4 and A6 sensors. The reference configuration for A4 (outer vessel normal field inductive) and A6 (outer vessel normal field steady state) sensors [9] are depicted in Fig. 3. Their main characteristics, taken from [9], are reported in Table 3.A for inductive probes (A4) and in Table 3.B for steady state probes (A6).

Using the same procedure described in the case of primary sensors only, 700 cases from test set were used to assess reconstruction capability of combined sensors. According to [6], the weighted sum is normalized to average poloidal field rather that to toroidal field on the magnetic axis. Results are reported in Fig. 4, showing some performance improvement. The analyses reported in this section refers to frequency ranges compatible with all sensors involved (up to 10 $\mathrm{Hz}$, but excluding DC for A4 and combined sets, while DC can be included for A6 sensors alone).

\section{Conclusions}

The performed analyses showed that the plasmaless Error Field Coefficients estimation procedure from proposed sensors is feasible in theory, yet very sensitive to nuisances. Primary sensors alone show satisfactory performance for "large enough" error fields. Supplementary sensors provide additional information improving primary sensors performance. The use of supplementary sensors for calibration purposes must be carefully studied. Results may be improved using more sophisticated data processing;

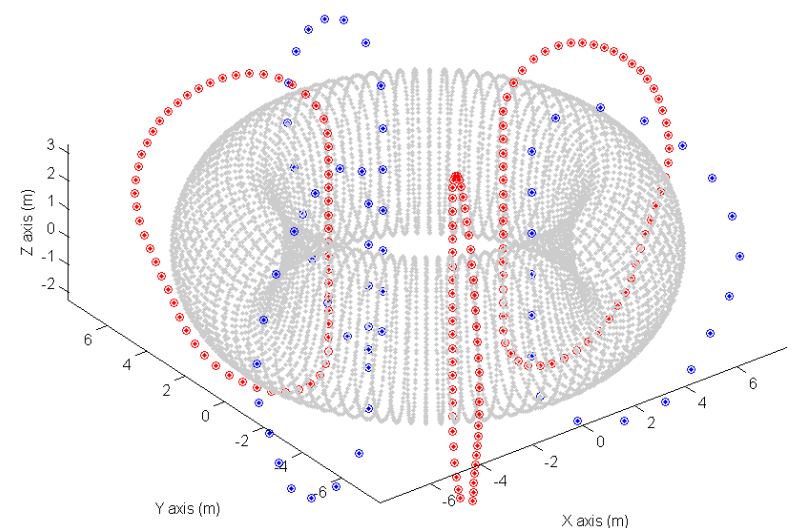

Fig. 3 - Outer vessel normal field sensors in this analysis (red: inductive probes, blue: steady state sensors). For the sake of comparison, also the equilibrium surface used for Fourier decomposition of field ( $\mathrm{q}=2$ at $\mathrm{SOF}$ of $15 \mathrm{MA}$ reference inductive scenario, grey points) is reported
TABLE 3.A KEY FIGURES FOR THE A4 SENSORS

\begin{tabular}{ll}
\hline \hline Number of Probes: & $\begin{array}{l}180 \text { Probes, on } 3 \text { sec- } \\
\text { tions, } 120^{\circ} \text { apart }\end{array}$ \\
\hline Total Sensor Error & Relative: $0.37 \%$, \\
\hline $\begin{array}{l}\text { Accuracy @ 2 } \sigma \text { for nor- } \\
\text { mal field measurement }\end{array}$ & Absolute: $4 \mathrm{mT}$ \\
\hline $\begin{array}{l}\text { Range for normal field } \\
\text { measurement }\end{array}$ & $0-2 \mathrm{~T}$ \\
\hline
\end{tabular}

TABLE 3.B KEY FIGURES FOR THE A6 SENSORS

\begin{tabular}{ll}
\hline \hline Number of Probes: & $\begin{array}{l}60 \text { Probes, on } 3 \text { sections, } \\
120^{\circ} \text { apart }\end{array}$ \\
\hline Total Sensor Error & Relative: $0.32 \%$, \\
\hline $\begin{array}{l}\text { Accuracy @2 } \sigma \text { for nor- } \\
\text { mal field measurement } \\
\text { Range for normal field } \\
\text { measurement }\end{array}$ & Absolute: $4 \mathrm{mT}$ \\
\hline \hline
\end{tabular}




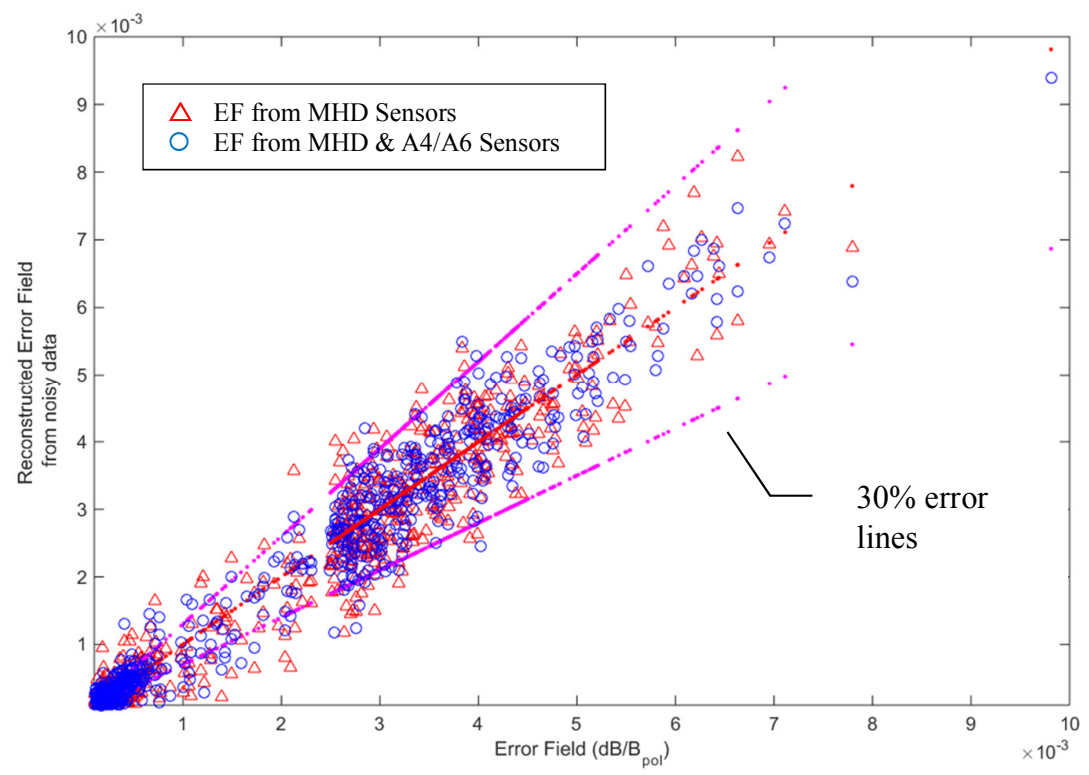

Fig. 4 - Comparison between "numerically simulated" and estimated EF amplitudes for test set. Magenta lines represent $+/-30 \%$ error levels. Signals from primary and supplementary sensors are considered.

\section{Acknowledgements}

The authors are thankful to the fruitful discussions held with R. Albanese, G. Ambrosino, A. Pironti, and F. Villone from C.R.E.A.T.E. The work reported was funded partially by Fusion for Energy under Grant F4E-GRT-047. This publication reflects the views of the authors only. Fusion for Energy cannot be held responsible for any use which may be made of the information contained herein. The views and opinions expressed herein do not necessarily reflect those of the ITER Organization

\section{References}

[1] R.J. La Haye et al. (1992), "Critical error fields for locked mode instability in Tokamaks", Phys. Fluids B, V.4, pp. 2098-2103.

[2] J.-K. Park, M. J. Schaffer, J. E. Menard, and A. H. Boozer (2007), “Control of Asymmetric Magnetic Perturbations in Tokamaks “, Phys. Rev. Lett. 99, 195003.

[3] J. Knaster et al. (2011), ITER non-axisymmetric error fields induced by its magnet system, Fus. Eng. Des., Vol. 86/6-8, pages 1053-1056.

[4] LaHaye, R. J., (1997), "Physics of locked modes in ITER: Error field limits, rotation for obviation, and measurement of field errors," General Atomics report, GA-A22468.

[5] G. Vayakis (2011), “Detailed functions of the Magnetics”, ITER Doc. D359V69.

[6] AA. VV. (2014), “Project Requirements”, ITER Doc. 27ZRW8.

[7] Abdi. H., \& Williams, L.J. (2010). "Principal component analysis.". Wiley Interdisciplinary Reviews: Computational Statistics, 2: 433-459.

[8] Cook, R. D. and Weisberg, S. (1982); Residuals and influence in regression, New York, NY: Chapman \& Hall. 
Error Field in Tokamaks: a Plasmaless Measurement Approach

A.G. Chiariello, A. Formisano, R. Martone, S. Arshad, G. Vayakis

[9] AA. VV. (2014), “System Design Description (DDD): Magnetic Diagnostics”, ITER Doc. 3UYQGX. 